\title{
New MDS Euclidean and Hermitian Self-Dual Codes over Finite Fields
}

\author{
Hongxi Tong, Xiaoqing Wang \\ Department of Mathematics, Shanghai University, Shanghai, China \\ Email: tonghx@shu.edu.cn, 2625453656@qq.com
}

How to cite this paper: Tong, H.X. and Wang, X.Q. (2017) New MDS Euclidean and Hermitian Self-Dual Codes over Finite Fields. Advances in Pure Mathematics, 7, 325-333.

https://doi.org/10.4236/apm.2017.75019

Received: April 12, 2017

Accepted: May 7, 2017

Published: May 10, 2017

Copyright $\odot 2017$ by authors and Scientific Research Publishing Inc. This work is licensed under the Creative Commons Attribution International License (CC BY 4.0).

http://creativecommons.org/licenses/by/4.0/

(c) (i) Open Access

\begin{abstract}
In this paper, we construct MDS Euclidean self-dual codes which are extended cyclic duadic codes. And we obtain many new MDS Euclidean self-dual codes. We also construct MDS Hermitian self-dual codes from generalized ReedSolomon codes and constacyclic codes.
\end{abstract}

\section{Keywords}

MDS Euclidean Self-Dual Codes, MDS Hermitian Self-Dual Codes, Constacyclic Codes, Cyclic Duadic Codes, Generalized Reed-Solomon Codes

\section{Introduction}

Let $\mathbb{F}_{q}$ denote a finite field with $q$ elements. An $[n, k, d]$ linear code $C$ over $\mathbb{F}_{q}$ is a $k$-dimensional subspace of $\mathbb{F}_{q}^{n}$. These parameters $n, k$ and $d$ satisfy $d \leq n-k+1$. If $d=n-k+1, C$ is called a maximum distance separable (MDS) code. MDS codes are of practical and theoretical importance. For examples, MDS codes are related to geometric objects called $n$-arcs.

The Euclidean dual code $C^{\perp}$ of $C$ is defined as

$$
C^{\perp}:=\left\{x \in \mathbb{F}_{q}^{n}: \sum_{i=1}^{n} x_{i} y_{i}=0, \forall y \in C\right\} .
$$

If $q=r^{2}$, the Hermitian dual code $C^{\perp H}$ of $C$ is defined as

$$
C^{\perp H}:=\left\{x \in \mathbb{F}_{r^{2}}^{n}: \sum_{i=1}^{n} x_{i} y_{i}^{r}=0, \forall y \in C\right\} .
$$

If $C$ satisfies $C=C^{\perp}$ or $C=C^{\perp H}, C$ is called Euclidean self-dual or Hermitian self-dual, respectively. In [1] [2] discussing Euclidean self-dual codes or Hermitian self-dual codes. If $C$ is MDS and Euclidean self-dual or Hermitian self-dual, $C$ is called an MDS Euclidean self-dual code or an MDS Hermitian self-dual code, respectively. In recent years, In [2]-[9] study the MDS self-dual 
codes. One of these problems in this topic is to determine existence of MDS self-dual codes. When $2 \mid q$, Grassl and Gulliver completely solve the existence of MDS Euclidean self-dual codes in [5]. In [6], Guenda obtain some new MDS Euclidean self-dual codes and MDS Hermitian self-dual codes. In [8], Jin and Xing obtain some new MDS Euclidean self-dual codes from generalized ReedSolomon codes.

In this paper, we obtain some new Euclidean self-dual codes by studying the solution of an equation in $\mathbb{F}_{q}$. And we generalize Jin and Xing's results to MDS Hermitian self-dual codes. We also construct MDS Hermitian self-dual codes from constacyclic codes. We discuss MDS Hermitian self-dual codes obtained from extended cyclic duadic codes and obtain some new MDS Hermitian self-dual codes.

\section{MDS Euclidean Self-Dual Codes}

A cyclic code $C$ of length $n$ over $\mathbb{F}_{q}$ can be considered as an ideal, $\langle g(x)\rangle$, of the ring $R=\frac{\mathbb{F}_{q}[x]}{x^{n}-1}$, where $g(x) \mid x^{n}-1$ and $(n, q)=1$. The set $T=\left\{0 \leq i \leq n-1 \mid g\left(\alpha^{i}\right)=0\right\}$ is called the defining set of $C$, where ord $\alpha=n$.

Let $S_{1}$ and $S_{2}$ be unions of cyclotomic classes modulo $n$, such that $S_{1} \cap S_{2}=\varnothing$ and $S_{1} \cup S_{2}=\mathbb{Z}_{n} \backslash\{0\}$ and $a S_{i}(\bmod n)=S_{i+1(\bmod 2)}$. Then the triple $\mu_{a}, S_{1}$ and $S_{2}$ is called a splitting modulo $n$. Odd-like codes $D_{1}$ and $D_{2}$ are cyclic codes over $\mathbb{F}_{q}$ with defining sets $S_{1}$ and $S_{2}$, respectively. $D_{1}$ and $D_{2}$ can be denoted by $\mu_{a}\left(D_{i}\right)=D_{i+1(\bmod 2)}$. Even-like duadic codes $C_{1}$ and $C_{2}$ are cyclic codes over $\mathbb{F}_{q}$ with defining sets $\{0\} \cup S_{1}$ and $\{0\} \cup S_{2}$, respectively. Obviously, $\mu_{a}\left(C_{i}\right)=C_{i+1(\bmod 2)}$. In [10], A duadic code of length $n$ over $\mathbb{F}_{q}$ exists if and only if $q$ is a quadratic residue modulo $n$.

Let $n \mid q-1$ and $n$ be an odd integer. $D_{1}$ is a cyclic code with defining set $T=\left\{1,2, \cdots, \frac{n-1}{2}\right\}$. Then $D_{1}$ is an $\left[n, \frac{n+1}{2}, \frac{n+1}{2}\right]$ MDS code. Its dual $C_{1}=D_{1}^{\perp}$ is also cyclic with defining set $T \cup\{0\}$. There are a pair of odd-like duadic codes $D_{1}=C_{1}^{\perp}$ and $D_{2}=C_{2}^{\perp}$ and a pair of even-like duadic codes $C_{2}=\mu_{-1}\left(C_{1}\right)$.

Lemma 1 [6] Let $n \mid q-1$ and $n$ be an odd integer. There exists a pair of MDS codes $D_{1}$ and $D_{2}$ with parameters $\left[n, \frac{n+1}{2}, \frac{n+1}{2}\right]$, and $\mu_{-1}\left(D_{i}\right)=D_{i+1(\bmod 2)}$.

Lemma 2 [11] Let $D_{1}$ and $D_{2}$ be a pair of odd-like duadic codes of length $n$ over $\mathbb{F}_{q}, \mu_{-1}\left(D_{i}\right)=D_{i+1(\bmod 2)}$. Assume that

$$
1+\gamma^{2} n=0
$$

has a solution in $\mathbb{F}_{q}$. Let $\tilde{D}_{i}=\left\{\tilde{c} \mid c \in D_{i}\right\}$ for $1 \leq i \leq 2$ and $\tilde{c}=\left(c_{0}, c_{1}, \cdots, c_{n-1}, c_{\infty}\right)$ with $c_{\infty}=-\gamma \sum_{i=0}^{n-1} c_{i}$. Then $\tilde{D}_{1}$ and $\tilde{D}_{2}$ are Euclidean self-dual codes. 
In [11], the solution of $\left(^{*}\right)$ is discussed when $n$ is an odd prime. In [5], the solution of $\left(^{*}\right)$ is discussed when $n$ is an odd prime power. Next, we discuss the solution of $\left(^{*}\right)$ for any odd integer $n$ with $n \mid q-1$.

Definition 1 (Legendre Symbol) [12] Let $p$ be an prime and $a$ be an integer.

$$
\left(\frac{a}{p}\right)= \begin{cases}0, & \text { if } a \equiv 0(\bmod p), \\ 1, & \text { if } a(\neq 0) \text { is a quadratic residue modulo } p \\ -1, & \text { if } a \text { is not a quadratic residue modulo } p\end{cases}
$$

\section{Proposition 1 [12]}

$$
\left(\frac{a}{p}\right)=\left(\frac{p_{1}}{p}\right) \cdots\left(\frac{p_{s}}{p}\right)
$$

where $a=p_{1} \cdots p_{s}$.

Definition 2 (Jacobi Symbol) [12] Let $m$ and $n(\neq 0)$ be two integers.

$$
\left(\frac{m}{n}\right)=\left(\frac{m}{p_{1}}\right) \cdots\left(\frac{m}{p_{h}}\right) \text {, }
$$

where $n=p_{1} \cdots p_{h}$.

We cannot obtain $m(\neq 0)$ is a quadratic residue modulo $n$ from $\left(\frac{m}{n}\right)=1$. But we have the next proposition.

Proposition 2 Let $m(\neq 0)$ and $n$ be two integers and $(m, n)=1$. If $m$ is a quadratic residue modulo $n$, then

$$
\left(\frac{m}{n}\right)=1
$$

If

$$
\left(\frac{m}{n}\right)=-1
$$

then $m$ is not a quadratic residue modulo $n$.

Proof Obviously.

Lemma 3 (Law of Quadratic Reciprocity) [12] Let $p$ and $r$ be odd primes, $(p, r)=1$.

$$
\left(\frac{p}{r}\right)\left(\frac{r}{p}\right)=(-1)^{\frac{r-1}{2} \cdot \frac{p-1}{2}} .
$$

Corollary 1 Let $p$ and $r$ be odd primes.

(1) When $p \equiv 1(\bmod 4)$ or $r \equiv 1(\bmod 4)$,

$$
\left(\frac{p}{r}\right)=\left(\frac{r}{p}\right) \text {. }
$$

(2) When $p \equiv r \equiv 3(\bmod 4)$,

$$
\left(\frac{p}{r}\right)=-\left(\frac{r}{p}\right) .
$$

Theorem 1 Let $q=r^{t}$ and $r$ be an odd prime. Let $n \mid q-1$ and $n$ be an odd integer. And 


$$
n=p_{1}^{e_{1}} \cdots p_{s}^{e_{s}} p_{s+1}^{e_{s+1}} \cdots p_{h}^{e_{h}}
$$

where

$$
p_{1} \equiv \cdots \equiv p_{s} \equiv 3(\bmod 4), \quad p_{s+1} \equiv \cdots \equiv p_{h} \equiv 1(\bmod 4) \text {. }
$$

(1) When $q \equiv 1(\bmod 4)$, there is a solution to $\left.{ }^{*}\right)$ in $\mathbb{F}_{q}$.

(2) Let $q \equiv 3(\bmod 4)$. If $\sum_{i=1}^{s} e_{i}$ is an odd integer, there is a solution to $\left.{ }^{*}\right)$ in $\mathbb{F}_{q}$.

Proof $(1) \quad q \equiv 1(\bmod 4)$.

(1.1) $r \equiv 3(\bmod 4)$. So we have that $t$ is even. Then every quadratic equation with coefficients in $\mathbb{F}_{r}$, such as Eq. $\left.{ }^{*}\right)$, has a solution in $\mathbb{F}_{r^{2}} \subseteq \mathbb{F}_{q}$.

(1.2) $r \equiv 1(\bmod 4)$ and $2 \mid t$. The proof is similar as (1.1).

(1.3) $r \equiv 1(\bmod 4)$ and $2 \nmid t$.

$$
1=\left(\frac{q}{n}\right)=\left(\frac{r}{n}\right)=\left(\frac{r}{p_{1}}\right)^{e_{1}} \cdots\left(\frac{r}{p_{h}}\right)^{e_{h}}=\left(\frac{p_{1}}{r}\right)^{e_{1}} \cdots\left(\frac{p_{h}}{r}\right)^{e_{h}}=\left(\frac{n}{r}\right) .
$$

So $n$ is a quadratic residue modulo $r$. And -1 is a quadratic residue modulo $r$. So there is a solution to $\left.{ }^{*}\right)$ in $\mathbb{F}_{q}$.

(2) $q \equiv 3(\bmod 4)$. Then $r \equiv 3(\bmod 4)$ and $t$ is odd.

$$
\begin{aligned}
1 & =\left(\frac{q}{n}\right)=\left(\frac{r}{n}\right)=\left(\frac{r}{p_{1}}\right)^{e_{1}} \cdots\left(\frac{r}{p_{s}}\right)^{e_{s}}\left(\frac{r}{p_{s+1}}\right)^{e_{s+1}} \cdots\left(\frac{r}{p_{h}}\right)^{e_{h}} \\
& =(-1)^{e_{1}}\left(\frac{p_{1}}{r}\right)^{e_{1}} \cdots(-1)^{e_{s}}\left(\frac{p_{s}}{r}\right)^{e_{s}}\left(\frac{p_{s+1}}{r}\right)^{e_{s+1}} \cdots\left(\frac{p_{h}}{r}\right)^{e_{h}} \\
& =(-1)^{\sum_{i=1}^{s} e_{i}}\left(\frac{p_{1}}{r}\right)^{e_{1}} \cdots\left(\frac{p_{s}}{r}\right)^{e_{s}}\left(\frac{p_{s+1}}{r}\right)^{e_{s+1}} \cdots\left(\frac{p_{h}}{r}\right)^{e_{h}}=(-1)^{\sum_{i=1}^{s} e_{i}}\left(\frac{n}{r}\right) .
\end{aligned}
$$

If $\sum_{i=1}^{s} e_{i}$ is odd, $n$ is not a quadratic residue modulo $r$. And -1 is not a quadratic residue modulo $r$. So $-n$ is a quadratic residue modulo $r$. There is a solution to $\left.{ }^{*}\right)$ in $\mathbb{F}_{q}$.

Remark In fact, $n \mid q-1$, and $n$ is an odd integer and $q \equiv 3(\bmod 4)$. We can easily prove that there is a solution to $\left(^{*}\right)$ in $\mathbb{F}_{q}$ if and only if $\sum_{i=1}^{s} e_{i}$ is an odd integer.

Let $n \mid q-1, \quad q \equiv 1(\bmod n) . \quad q$ is a quadratic residue modulo $n$. $y^{2} \equiv q(\bmod n)$. Let $q=r^{t}$ and $q \equiv 3(\bmod 4)$, where $r$ is a prime. Then $r \equiv 3(\bmod 4)$ and $t$ is odd. Equation $\left.{ }^{*}\right)$ has solutions in $\mathbb{F}_{q}$ if and only if Equation $\left.{ }^{*}\right)$ has solutions in $\mathbb{F}_{r}$. And $r$ is a quadratic residue modulo $n$. $\left(y r^{-\frac{t-1}{2}}\right)^{2} \equiv r(\bmod n)$. Let $p$ be an odd prime divisor of $n . r$ is a quadratic residue modulo $p$. Then $\left(\frac{r}{p}\right)=1$. By Law of Quadratic Reciprocity, $p \mid n$,

$$
\left(\frac{p}{r}\right)=\left\{\begin{array}{ll}
1, & p \equiv 1(\bmod 4) \\
-1, & p \equiv 3(\bmod 4)
\end{array}\right. \text {. }
$$

The Legendre symbol 


$$
\begin{aligned}
\left(\frac{-n}{r}\right) & =\left(\frac{-1}{r}\right)\left(\frac{p_{1}}{r}\right)^{e_{1}} \cdots\left(\frac{p_{s}}{r}\right)^{e_{s}}\left(\frac{p_{s+1}}{r}\right)^{e_{s+1}} \cdots\left(\frac{p_{h}}{r}\right)^{e_{h}} \\
& =(-1)^{1+\sum_{i=1}^{s} e_{i}}=\left\{\begin{array}{ll}
1, & \sum_{i=1}^{s} e_{i} \text { is odd } \\
-1, & \sum_{i=1}^{s} e_{i} \text { is even }
\end{array},\right.
\end{aligned}
$$

where $n=p_{1}^{e_{1}} \cdots p_{s}^{e_{s}} p_{s+1}^{e_{s+1}} \cdots p_{h}^{e_{h}}, \quad p_{1} \equiv \cdots \equiv p_{s} \equiv 3(\bmod 4)$ and $p_{s+1} \equiv \cdots \equiv p_{h} \equiv 1(\bmod 4)$.

Theorem 2 Let $q=r^{t}$ be a prime power, $n \mid q-1$ and $n$ be an odd integer. Then there exists a pair $D_{1}, D_{2}$ of MDS odd-like duadic codes of length $n$ and $\mu_{-1}\left(D_{i}\right)=D_{i+1(\bmod 2)}$, where even-like duadic codes are MDS self-orthogonal, and $T_{1}=\left\{1, \cdots, \frac{n-1}{2}\right\}$. Furthermore,

(1) If $q=2^{t}$, then $\tilde{D}_{i}$ are $\left[n+1, \frac{n+1}{2}, \frac{n+3}{2}\right]$ MDS Euclidean self-dual codes.

(2) If $q \equiv 1(\bmod 4)$, then $\tilde{D}_{i}$ are $\left[n+1, \frac{n+1}{2}, \frac{n+3}{2}\right]$ MDS Euclidean self-dual codes.

(3) If $q \equiv 3(\bmod 4)$ and $\sum_{i=1}^{s} e_{i}$ is an odd integer, then $\tilde{D}_{i}$ are $\left[n+1, \frac{n+1}{2}, \frac{n+3}{2}\right]$ MDS Euclidean self-dual codes, where $n=p_{1}^{e_{1}} \cdots p_{s}^{e_{s}} p_{s+1}^{e_{s+1}} \cdots p_{t}^{e_{h}}$ and $p_{1} \equiv \cdots \equiv p_{s} \equiv 3(\bmod 4)$, $p_{s+1} \equiv \cdots \equiv p_{h} \equiv 1(\bmod 4)$.

Proof Obviously, $D_{i}$ are $\left[n, \frac{n+1}{2}, \frac{n+1}{2}\right]$ MDS odd-like duadic codes. If there is a solution to $\left(^{*}\right)$, we want to prove $\tilde{D}_{i}$ are $\left[n+1, \frac{n+1}{2}, \frac{n+3}{2}\right]$ MDS Euclidean self-dual codes, and we only need to prove that

$$
c \in D_{i} \text { and } w t(c)=\frac{n+1}{2} \text {, then } w t(\tilde{c})=\frac{n+1}{2}+1 .
$$

This is equivalent to prove that $c_{\infty} \neq 0$. It can be proved similarly by which proved in [5].

When $q=2^{t}$, there is a solution to $\left(^{*}\right)$ in $\mathbb{F}_{2^{t}}, \tilde{D}_{i}$ are $\left[n+1, \frac{n+1}{2}, \frac{n+3}{2}\right]$ MDS Euclidean self-dual codes by Lemma 2.

We can obtain (2) and (3) from Theorem 1 and Lemma 2. Theorem 2 is proved.

We list some new MDS Euclidean self-dual codes in the next Table 1.

\section{MDS Hermitian Self-Dual Codes}

Let $n \leq q^{2}$. We choose $n$ distinct elements $\left\{\alpha_{1}, \cdots, \alpha_{n}\right\}$ from $\mathbb{F}_{q^{2}}$ and $n$ nonzero elements $\left\{v_{1}, \cdots, v_{n}\right\}$ from $\mathbb{F}_{q^{2}}$. The generalized Reed-Solomon code 
Table 1. Some new MDS Euclidean self-dual codes.

\begin{tabular}{cc}
\hline $\mathrm{n}$ & $\mathrm{q}$ \\
4 & $2^{2}, 7$ \\
6 & $2^{4}, 3^{4}$ \\
8 & $2^{3}, 3^{6}$ \\
10 & $2^{6}, 5^{6}$ \\
12 & $3^{5}$ \\
14 & $2^{12}, 3^{6}$ \\
16 & $31,31^{2}, 31^{3}$ \\
18 & $3^{16}$ \\
20 & $5^{9}$ \\
22 & $5^{6}$ \\
24 & $3^{11}$ \\
26 & $7^{4}$ \\
28 & $7^{9}$ \\
30 & 59 \\
156 & $5^{4}$ \\
&
\end{tabular}

$$
\operatorname{GRS}_{k}(\alpha, v):=\left\{\left(v_{1} f\left(\alpha_{1}\right), \cdots, v_{n} f\left(\alpha_{n}\right)\right): f(x) \in \mathbb{F}_{q^{2}}[x], \operatorname{deg} f(x) \leq k-1\right\}
$$

is a $q^{2}$-ary $[n, k, n-k+1]$ MDS code, where $\alpha=\left(\alpha_{1}, \cdots, \alpha_{n}\right)$ and $v=\left(v_{1}, \cdots, v_{n}\right)$.

Theorem 3 Let $n \leq q$ and $2 \mid n$. Let $\left\{\alpha_{1}, \cdots, \alpha_{n}\right\}$ be $n$ distinct elements from $\mathbb{F}_{q}\left(\subseteq \mathbb{F}_{q^{2}}\right)$ and $u_{i}=\prod_{1 \leq j \leq n, j \neq i}\left(\alpha_{i}-\alpha_{j}\right)^{-1}, 1 \leq i \leq n$. Then there exist $v_{i} \in \mathbb{F}_{q^{2}}$ such that $u_{i}=v_{i}^{2}$, for $i=1, \cdots, n$, and the generalized Reed-Solomon code $\operatorname{GRS}_{\frac{n}{2}}(\alpha, v)$ is an $\left[n, \frac{n}{2}, \frac{n}{2}+1\right]$ MDS Hermitian self-dual code over $\mathbb{F}_{q^{2}}$, where $\alpha=\left(\alpha_{1}, \cdots, \alpha_{n}\right)$ and $v=\left(v_{1}, \cdots, v_{n}\right)$.

Proof Obviously, $u_{i}(\neq 0) \in \mathbb{F}_{q}\left(\subseteq \mathbb{F}_{q^{2}}\right)$ for $1 \leq i \leq n$. So there exist $v_{i}(\neq 0) \in \mathbb{F}_{q^{2}}$ such that $u_{i}=v_{i}^{2}$ for $1 \leq i \leq n$. The generalized Reed-Solomon code $\operatorname{GRS}_{\frac{n}{2}}(\alpha, v)$ is an $\left[n, \frac{n}{2}, \frac{n}{2}+1\right]$ MDS code over $\mathbb{F}_{q^{2}}$. For proving the generalized Reed-Solomon code $G R S_{\frac{n}{2}}(\alpha, v)$ is Hermitian self-dual over $\mathbb{F}_{q^{2}}$, we only prove

$$
\left(v_{1} \alpha_{1}^{l}, \cdots, v_{n} \alpha_{n}^{l}\right) \cdot\left(v_{1}^{q} \alpha_{1}^{k q}, \cdots, v_{n}^{q} \alpha_{n}^{k q}\right)=0,0 \leq l, k \leq \frac{n}{2}-1 .
$$

From the choose of $\alpha_{i}, v_{i}$ and [8, Corollary 2.3],

$$
\begin{aligned}
& \left(v_{1} \alpha_{1}^{l}, \cdots, v_{n} \alpha_{n}^{l}\right) \cdot\left(v_{1}^{q} \alpha_{1}^{k q}, \cdots, v_{n}^{q} \alpha_{n}^{k q}\right) \\
& =\left(v_{1} \alpha_{1}^{l}, \cdots, v_{n} \alpha_{n}^{l}\right) \cdot\left(v_{1} \alpha_{1}^{k}, \cdots, v_{n} \alpha_{n}^{k}\right)=0,0 \leq l, k \leq \frac{n}{2}-1 .
\end{aligned}
$$


So the generalized Reed-Solomon code $\operatorname{GRS}_{\frac{n}{2}}(\alpha, v)$ is an $\left[n, \frac{n}{2}, \frac{n}{2}+1\right]$ MDS Hermitian self-dual code over $\mathbb{F}_{q^{2}}$.

Next we construct MDS Hermitian self-dual codes from constacyclic codes.

Let $C$ be an $[n, k] \lambda$-constacyclic code over $\mathbb{F}_{q^{2}}$ and $(n, q)=1 . C$ is considered as an ideal, $\langle g(x)\rangle$, of $\frac{F_{q^{2}}[x]}{x^{n}-\lambda}$, where $g(x) \mid\left(x^{n}-\lambda\right)$. Simply, $C=\langle g(x)\rangle$.

Lemma 4 [2] Let $\lambda \in \mathbb{F}_{q^{2}}^{*}, \quad r=\operatorname{ord}_{q^{2}}(\lambda)$, and $C$ be a $\lambda$-constacyclic code over $\mathbb{F}_{q^{2}}$. If $C$ is Hermitian self-dual, then $r \mid q+1$.

Lemma 5 [2] Let $n=2^{a} n^{\prime} \quad(a>0)$ and $r=2^{b} r^{\prime}$ be integers such that $2 \nmid n^{\prime}$ and $2 \nmid r^{\prime}$. Let $q$ be an odd prime power such that $(n, q)=1$ and $r \mid q+1$, and let $\lambda \in \mathbb{F}_{q^{2}}$ has order $r$. Then Hermitian self-dual $\lambda$-constacyclic codes over $\mathbb{F}_{q^{2}}$ of length $n$ exist if and only if $b>0$ and $q \neq \equiv-1\left(\bmod 2^{a+b}\right)$.

Let $r=\operatorname{ord}_{q^{2}}(\lambda)$ and $r \mid q+1$.

$$
O_{r, n}=\{1+r j \mid j=0,1, \cdots, n-1\} .
$$

Then $\alpha^{i}\left(i \in O_{r, n}\right)$ are all solutions of $x^{n}-\lambda=0$ in some extension field of $\mathbb{F}_{q^{2}}$, where ord $\alpha=r n$. $C$ is called a $\lambda$-constacyclic code with defining set $T \subseteq O_{r, n}$, if

$$
C=\langle g(x)\rangle \text { and } g\left(\alpha^{i}\right)=0, \forall i \in T .
$$

Theorem 4 Let $n=2^{a} n^{\prime}(a>0)$ and $r=2^{b} r^{\prime}(b>0) . \quad r n \mid q^{2}-1 . \quad \lambda \in \mathbb{F}_{q^{2}}^{*}$ with $\operatorname{ord} \lambda=r . q \neq \equiv-1\left(\bmod 2^{a+b}\right)$. If $r n \mid 2(q+1)$, there exists an MDS Hermitian self-dual code $C$ over $\mathbb{F}_{q^{2}}$ with length $n, C$ is a $\lambda$-constacyclic code with defining set

$$
T=\left\{1+r j \mid 0 \leq j \leq \frac{n}{2}-1\right\} .
$$

Proof If $r n \mid q^{2}-1, C_{q^{2}}(i)=\{i\}$, for $i \in O_{r, n}$, where $C_{q^{2}}(i)$ denote the $q^{2}$-cyclotomic coset of $i \bmod r n$. And $|T|=\frac{n}{2}, C$ is an $\left[n, \frac{n}{2}, \frac{n}{2}+1\right]$ MDS $\lambda$-constacyclic code by the BCH bound of constacyclic code.

When $r n \mid 2(q+1), q=\frac{r n l}{2}-1$. Because $q \not \equiv-1\left(\bmod 2^{a+b}\right), l$ is odd.

$$
(-q)(1+r j)=-q-q r j \equiv 1-\frac{r n l}{2}+r j \equiv 1+r\left(\frac{n}{2}+j\right)(\bmod r n) .
$$

So

$$
(-q) T \cap T=\varnothing .
$$

$C$ is MDS Hermitian self-dual by the relationship of roots of a constacyclic code and its Hermitian dual code's roots.

Remark The MDS Hermitian self-dual constacyclic code obtained from Theorem 4 is different with the MDS Hermitian self-dual constacyclic code in 
[12], because $(q+1, q-1)=2$ for an odd prime power $q$. If $r=2, C$ is negacyclic. Theorem 4 can be stated as follow.

Corollary 2 Let $n=2^{a} n^{\prime}(a \geq 1)$ and $n^{\prime}$ is odd. Let

$$
q \equiv-1\left(\bmod 2^{a} n^{\prime \prime}\right) \text { and } q \equiv 2^{a}-1\left(\bmod 2^{a+1}\right),
$$

where $n^{\prime} \mid n^{\prime \prime}$ and $n^{\prime \prime}$ is odd. Then there exists an MDS Hermitian self-dual code $C$ of length $n$ which is negacyclic with defining set

$$
T=\left\{1+2 j \mid j=0,1, \cdots, \frac{n}{2}-1\right\} .
$$

Especially, when $a=1$, Corollary 2 is similar as [5, Theorem 11].

From Theorem 3 and Theorem 4, we obtain the next theorem.

Theorem 5 Let $n \leq q+1$ and $n$ be even. There exists an MDS Hermitian self-dual code with length $n$ over $\mathbb{F}_{q^{2}}$.

\section{Conclusion}

In this paper, we obtain many new MDS Euclidean self-dual codes by solving the Equation $\left.{ }^{*}\right)$ in $\mathbb{F}_{q}$. We generalize the work of [8] to MDS Hermitian self-dual codes, and we construct new MDS Hermitian self-dual codes from constacyclic codes. We obtain that there exists an MDS Hermitian self-dual code with length $n$ over $\mathbb{F}_{q^{2}}$, where $n \leq q+1$ and $n$ is even. And we also discuss these MDS Hermitian self-dual codes, which are extended cyclic duadic codes. Some new MDS Hermitian self-dual codes are obtained.

\section{References}

[1] Dicuangco, L., Moree, P. and Solé, P. (2007) The Lengths of Hermitian Self-Dual Extended Duadic Codes. Journal of Pure and Applied Algebra, 209, 223-237. https://doi.org/10.1016/j.jpaa.2006.05.024

[2] Yang, Y.S. and Cai, W.C. (2015) On Self-Dual Constacyclic Codes over Finite Fields. Designs, Codes and Cryptography, 74, 355-364.

https://doi.org/10.1007/s10623-013-9865-9

[3] Aaron Gulliver, T., Kim, J.L. and Lee, Y. (2008) New MDS or Near-MDS Self-Dual Codes. IEEE Transactions on Information Theory, 54, 4354-4360.

https://doi.org/10.1109/TIT.2008.928297

[4] Georgiou, S. and Koukouvinos, C. (2002) MDS Self-Dual Codes over Large Prime Fields. Finite Fields and Their Applications, 8, 455-470. https://doi.org/10.1016/S1071-5797(02)90353-9

[5] Grassel, M. and Aaron Gulliver, T. (2008) On Self-Dual MDS Codes. Proceedings of ISIT, Barcelona, 2008, 1954-1957.

[6] Guenda, K. (2012) New MDS Self-Dual Codes over Finite Fields. Designs, Codes and Cryptography, 62, 31-42. https://doi.org/10.1007/s10623-011-9489-x

[7] Harada, M. and Kharaghani, H. (2008) Orthogonal Designs and MDS Self-Dual Codes. The Australasian Journal of Combinatorics, 35, 57-67.

[8] Jin, L.F. and Xing, C.P. (2016) New MDS Self-Dual Codes from Generalized ReedSolomon Codes. Arxiv: 1601.04467v1

[9] Kim, J.L. and Lee, Y. (2004) Euclidean and Hermitian Self-Dual MDS Codes over Large Finite Fields. Journal of Combinatorial Theory, Series A, 105, 79-95. 
https://doi.org/10.1016/j.jcta.2003.10.003

[10] Smid, M.H.M. (1983) Duadic Codes. IEEE Transactions on Information Theory, 33, 432-433.

[11] Huffman, W.C. and Pless, V. (2003) Fundamentals of Erro-Correcting Codes. Cambridge University Press, Cambridge. https://doi.org/10.1017/CBO9780511807077

[12] Pang, C.D. and Pang, C.B. (2002) Elementary Number Theory. Beijing University Press, Beijing. (In Chinese)

Submit or recommend next manuscript to SCIRP and we will provide best service for you:

Accepting pre-submission inquiries through Email, Facebook, LinkedIn, Twitter, etc. A wide selection of journals (inclusive of 9 subjects, more than 200 journals) Providing 24-hour high-quality service User-friendly online submission system Fair and swift peer-review system Efficient typesetting and proofreading procedure Display of the result of downloads and visits, as well as the number of cited articles Maximum dissemination of your research work

Submit your manuscript at: http://papersubmission.scirp.org/ Or contact apm@scirp.org 